American SLE population have shown a higher prevalence of LN, higher severity, and less favorable outcomes (1).

Objectives: To determine the incidence of lupus nephritis and end-stage renal failure, as well as evaluate progression of renal function and proteinuria during an 18-month follow up in colombian patients with SLE.

Methods: A retrospective cohort study was conducted in 1448 patients with SLE, 41 of which were diagnosed with LN between August/2014 and July/2015. Follow up was made for 18 months, analyzing glomerular filtration rate (GFR) and proteinuria, induction and manteinance therapy, renal relapses, hospitalizations and mortality. Univariate analysis was done to describe sociodemographic and clinical variables. Longitudinal data analysis was performed using linear mixed models with random intercepts. In all cases, a $\mathrm{p}$ value $<0.05$ was considered statistically significant.

Results: Clinical characteristics of patients with LN are shown in table 1. Eightyfive percent of $L N s$ where biopsy-proven. Incidence of $L N$ was 2,83 cases/100 SLE patients/year. The incidence of end-stage renal failure was 7,31 cases/100 LN patients. During the 18 -month follow up, $34 \%$ of patients had hospitalizations related to SLE activity or complications, $7,3 \%$ renal relapse, $2,4 \%$ rebiopsy, and no mortality cases. Induction therapy was done with cyclophosphamide in $58,5 \%$ and with mycophenolate mofetil in $41,4 \%$, with $30 \%$ of the patients requiring re-induction therapy, Ten percent of patients required use of rituximab due to refractory response to multiple treatments. In longitudinal linear analyses, age at

Table 1. Clinical characteristics of patients with lupus nephritis

\begin{tabular}{lc}
\hline Characteristic (N=41) & Mean (SD) \\
\hline Age & $37,2(11,2)$ \\
Age at diagnosis & $32,2(11,1)$ \\
Years of evolution & $4,6(4,4)$ \\
& $\mathrm{N}(\%)$ \\
Female & $38(92,7)$ \\
Malar rash & $11(28,2)$ \\
Discoid lupus & $2(4,9)$ \\
Photosensitivity & $18(46,1)$ \\
Oral ulcers & $14(34,1)$ \\
Serositis & $16(44,4)$ \\
Arthritis & $31(75,6)$ \\
Neurological involvement & $5(12,2)$ \\
Hematological involvement & $32(78)$ \\
ANAs $(+)$ & $39 / 39(100)$ \\
Anti dsDNA $(+)$ & $29 / 41(70,7)$ \\
Anti Sm $(+)$ & $17 / 35(48,6)$ \\
Anti Ro $(+)$ & $19 / 36(52,7)$ \\
Anti La $(+)$ & $10 / 34(29,4)$ \\
Anti RNP $(+)$ & $18 / 36(50)$ \\
\hline
\end{tabular}

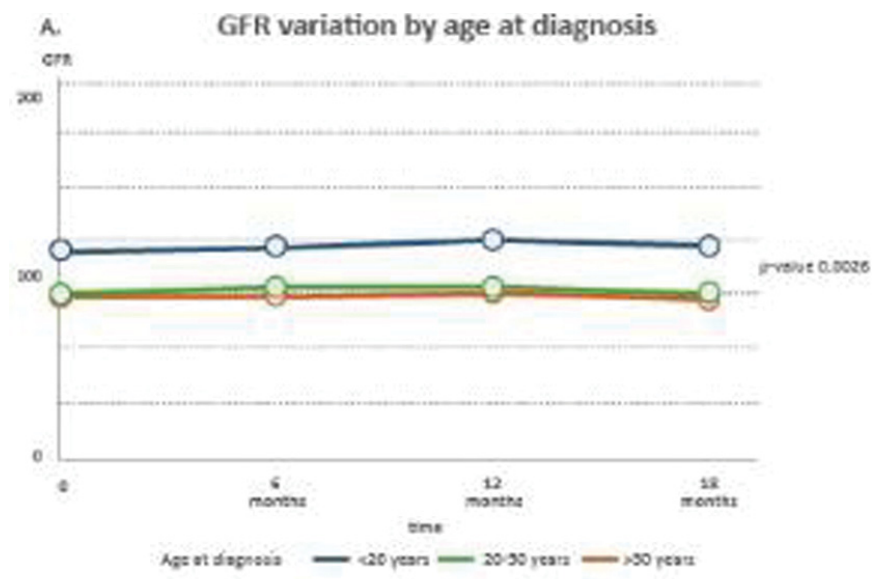

B. Proteinuria variation by biopsy classification

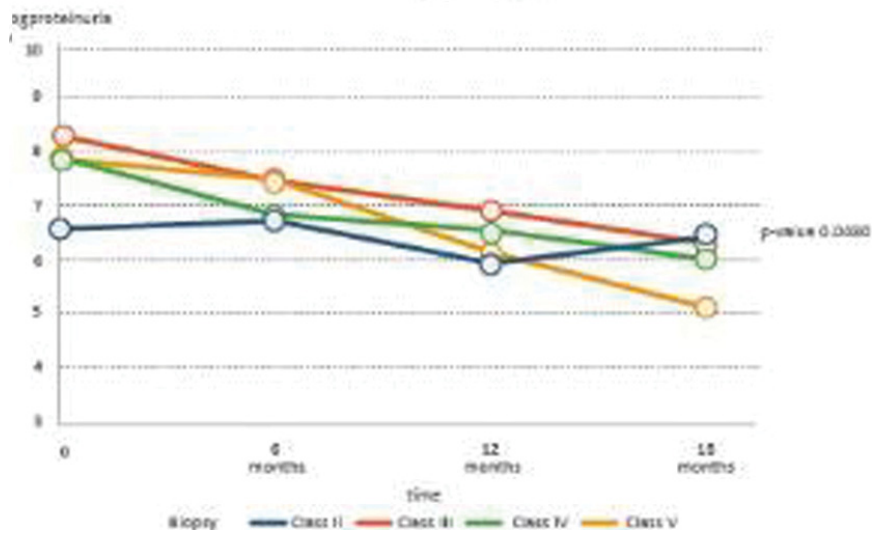

diagnosis and anti dsDNA was positively associated to GFR variations (Figure $1, \mathrm{~A})$, while anti Sm, hematologic involvement and biopsy classification were associated to proteinuria variations during time of follow-up (Figure 1,B).

Conclusions: In a real life scenario, anual incidence of lupus nephritis was 2,83 cases/100 SLE patients. A high proportion of patients with refractory response to multiple immunomodulatory treatments for $L N$ were identified.

References:

[1] Pons-Estel GJ, Catoggio LJ, Cardiel MH, Bonfa E, Caeiro F, Sato E, et al. Lupus in Latin-American patients: lessons from the GLADEL cohort. Lupus. 2015;24(6):536-45.

Disclosure of Interest: None declared

DOI: 10.1136/annrheumdis-2017-eular.6137

\section{FRI0288 NAILFOLD CAPILLAROSCOPIC PICTURES IN A COHORT OF UNDIFFERENTIATED CONNECTIVE TISSUE DISEASE (UCTD) PATIENTS AND IN THOSE THAT MOVE TO SYSTEMIC LUPUS ERYTHEMATOSUS}

M. Meroni ${ }^{1}, 2$, C. Pizzorni ${ }^{1}$, A. Sulli ${ }^{1}$, M. Limonta ${ }^{2}$, M. Cutolo ${ }^{1} .{ }^{1}$ Research Laboratory and Academic Division of Clinical Rheumatology, Internal Medicine Dept., University of Genova, Genova; ${ }^{2}$ Rheumatology Unit, ASST Papa Giovanni XXIII, Bergamo, Italy

Background: Nailfold capillaroscopy (NVC) is a useful, non-invasive, reproducible and cost-effective diagnostic tool, able to assess the shape of capillaries in the nailfold bed. According to the presence of peculiar abnormalities, it is essential in the early differential diagnosis of connective tissue diseases (CTDs), mainly "scleroderma-spectrum disorders" (SSD). Despite its large diffusion, no univocal NVC patterns have been ascribed to undifferentiated connective tissue disease (UCTD) as well as to systemic lupus erythematosus (SLE).

Objectives: The aim of the study was to evaluate the most common NVC pictures in a population of UCTD patients and if selected NVC pictures might be linked to SLE onset in these patients.

Methods: We evaluated a cohort of 42 UCTD-affected women, diagnosed according to 2014 criteria proposed by Mosca et al. (age, 38 years \pm 46 months; duration of disease, $71 \pm 54$ months) presenting Raynaud's phenomenon. During the observational period (3 years), all of the UCTD patients were evaluated every 6 months. We considered the following NVC parameters/pictures: presence of ectasic capillary loops (diameter $\geq 20 \mu \mathrm{m}$ ); giant capillaries (diameter $\geq 50 \mu \mathrm{m}$ ); hemosiderin deposits/microhemorrages; capillary number reduction; meandering capillaries (tortuosity); elongated capillaries; ramified/bushy capillaries; micro-vascular array disorganization. SLE diagnosis was posed according to the 2012 SLICC/ACR criteria. Qualitative variables were expressed in frequencies; their association, by non-parametric tests; quantitative variables, by analysis of co-variance. Results: Non-specific NVC alterations (for instance, not suggestive of SDD) were detected in $40(98 \%)$ of the UCTD patients during the observational period. On the other hands, the presence of hemosiderin deposits, ectasic loops, elongated and ramified capillaries was found associated to the clinical subgroup of UCTD patients that later developed SLE (4/42 subjects, $10 \%$; $O R=10.5)$. In particular, the independent variables "hemosiderin deposits/microhemorrages" (OR=8.32) and "elongated capillaries" (OR 6.28), were found significantly linked to the SLE onset $(p<0.05)$, whereas the independent variables "tortuosity" $(O R=12.16)$ and "ramified/bushy capillaries" (OR 9.47) were, at the opposite, predictive for the prosecution of the status of UCTD patient $(p<0.05)$.

Conclusions: The present study reports NVC pictures that can be more frequently observed in UCTD patients that include "tortuosity" and "ramified/bushy capillaries". In addition, the NVC analysis suggests the presence of typical capillaroscopic microvascular abnormalities, "hemosiderin deposits/microhemorrages" and "elongated capillaries", that more frequently seem observed in those UCTD patients that move to SLE onset.

References:

[1] Hughes M et al. Best Pract Res Clin Rheumatol. 2016;30:112-32.

[2] Ingegnoli F, et al. J Clin Rheumatol. 2005;11(6):295-8.

[3] Mosca M, et al. J Autoimmun. 2014:48-9:50-2.

[4] Cutolo M et al. Best Pract Res Clin Rheumatol. 2005;19(3):437-52.

Disclosure of Interest: None declared

DOI: 10.1136/annrheumdis-2017-eular.5742

\section{FRI0289 CEREBROVASCULAR DISEASE IN THE ANTIPHOSPHOLIPID SYNDROME}

M. Estévez, A. Argibay, L. Rodriguez, M. Freire, B. Gimena,

J. Fernández-Martín, A. Rivera. Systemic Autoimmune diseases and Thrombosis Unit, University Hospital Complex of Vigo, Vigo, Spain

Background: Antiphospholipid syndrome (APS) is a thrombophilic disorder characterized by recurrent arterial and venous thrombosis, and also pregnancy losses associated to antiphospholipid antibodies (APA). Cerebrovascular disease (CVD) is the most common and severe arterial thrombotic manifestation in patients with APS.

Objectives: 1.To determine the prevalence and the type of CVD in patients with APS. 2.To compare the recurrent strokes, affected brain areas, hospitalization, treatment and mortality between patients with CVD, with and without APS. 
Abstract FRI0289 - Tabe 1

\begin{tabular}{|c|c|c|c|c|c|c|c|c|c|c|}
\hline & Age & OAC & APT & Vascular risk factors & Valvular disease & Brain stem & Frontal lobe & Strokes $(\geq 2)$ & Mortality & Follow-up \\
\hline CVD with APS & $57,48 \pm 21,52$ & $48 \%$ & $20 \%$ & $80 \%$ & $20 \%$ & $0 \%$ & $12 \%$ & $44 \%$ & $40 \%$ & $8,64 \pm 6,72$ \\
\hline CVD without APS & $\begin{array}{c}61,16 \pm 20,6 \\
\text { p } 0,76\end{array}$ & $\begin{array}{c}0 \% \\
p<001\end{array}$ & $\begin{array}{c}72 \% \\
p<001\end{array}$ & $\begin{array}{c}68 \% \\
\text { p } 0,33\end{array}$ & $\begin{array}{c}0 \% \\
\text { p } 0,02\end{array}$ & $\begin{array}{l}20 \% \\
\text { p0,02 }\end{array}$ & $\begin{array}{c}36 \% \\
\text { p } 0,04\end{array}$ & $\begin{array}{c}16 \% \\
\text { p } 0.03\end{array}$ & $\begin{array}{c}44 \% \\
\text { p } 0,77\end{array}$ & $\begin{array}{c}2,04 \pm 2,99 \\
p<001\end{array}$ \\
\hline
\end{tabular}

Methods: Retrospective and, descriptive study of patients with APS (Sidney criteria) and CVD followed for a long period of time in a specific Systemic Autoimmune Diseases and Thrombosis Unit. Subsequently, retrospective casecontrol study was performed. Case definition: patients with CVD attributable to APS. Control definition: patients with CVD without APS. The controls were matched with cases by sex and age (within the same decade). Chi-square and t-student were used, using the statistical package SPSS22.0.

Results: 25 patients (25/88 28,4\%) had CVD, 19 (76\%) of primary APS and $6(21 \%)$ of secondary APS. 17 patients $(71,6 \%)$ were female. The mean age was $57,48 \pm 21,52$ [range $13-89$ ], with a mean follow-up of $8,64 \pm 6,72$ years. $24 \%$ of patients had atrial fibrillation, $80 \%$ had one cardiovascular risk factor and $48 \%$ had two or more factors (hypertension 68\%, hypercholesterolemia 36\%, diabetes $20 \%$, tabaquism $4 \%$ ). Echocardiographic study was performed in $72 \%$ of patients with APS. Mitral valve was mainly involved. Most CVD were ischemic events (92\%). The brain areas most involved were the basal ganglia (36\%), together with the parietal and temporal lobe (16\% respectively). $40 \%$ had two or more affected regions. $44 \%$ of the patients had two or more episodes of stroke. Lupus anticoagulant was positive in $40 \%$, anticardiolipin antibodies in $76 \%$ and anti- $\beta 2$ glycoproteinl antibodies in $20 \%$. No differences were found with isotypes of APA and recurrent thrombosis or mortality. The treatment applied was oral anticoagulants (OAC) $(48 \%)$, antiplatelet therapy (APT) $(20 \%)$ and low molecular weight heparin (20\%). In 10 patients (40\%) CVD was diagnosed before APS (mean $8.64 \pm 6.7$ years). The mortality was $44 \%$ and $40 \%$ of the patients were hospitalized more than once. When we compared the groups: treatment, performed echocardiogram, valvular disease, affected brain areas, recurrent strokes and follow-up time, revealed significant differences (see table 1).OAC were more used in the patients with APS and APT was the most common in control group. Valvular disease was more frequent in case group. The brainstem and the frontal lobe were the areas more affected in patients without APS. The number of strokes was higher in APS group. The patients with CVD and APS had a long-term follow-up.

Conclusions: The prevalence of CVD in our series of APS was $28.4 \%$ and most often were ischemic events. Most of the patients were women with high recurrent strokes and mortality. No differences were found with isotypes of APA and recurrent thrombosis or prognosis. CVD with APS patients had more recurrent strokes and longer follow-up.

Disclosure of Interest: None declared

DOI: 10.1136/annrheumdis-2017-eular.4509

\section{FRI0290 INTERLEUKIN 10 GENE POLYMORPHISMS IN PRIMARY SJÖGREN SYNDROME IN A TUNISIAN POPULATION}

M. Tougorti ${ }^{1}$, A. Kallel ${ }^{2}$, I. Ben Ghorbel ${ }^{1}$, R. Jemaa ${ }^{2}$, M.H. Houman ${ }^{1}$, N. Kaabachi ${ }^{2}$. ${ }^{1}$ Internal Medicine; ${ }^{2}$ Biochemistry, Rabta university hospital, Tunis, Tunisia

Background: Primary Sjögren syndrome (PSS) is one of the most common autoimmune systemic rheumatic diseases although its prevalence anging between 0.6 and $1.7 \%$. PSS affects exocrine glands and lead to sicca syndrome. Interleukin-10 (IL-10) is a pleiotropic cytokine that is involved in the inflammation process of PSS.

Objectives: The aim of our study was to determine in a Tunisian population, clinical and biological characteristics of patients with primary PSS, allelic and genotypic frequencies of $(-1082 \mathrm{G} / \mathrm{A},-819 \mathrm{C} / \mathrm{T}$ and $-592 \mathrm{C} / \mathrm{A})$ polymorphisms in IL-10 gene and to evaluate the association of these polymorphism with PSS.

Methods: The population we studied consisted of 242 subjects with female predominance (average age at diagnosis $=49$ years), divided into 84 PSS patients (fulfilling the revised AECG criteria 2002 and/or ACR proposed criteria 2012), recruited in the internal medicine department of the Rabta hospital and 158 controls recruited in the Greater Tunis. II 10 level was assessed by ELISA. Polymorphisms genotyping of the IL-10 gene was done using PCR-RFLP technique.

Results: I110 plasma level was lower in PSS patients $(23.71 \mathrm{pg} / \mathrm{ml}, \mathrm{n}=73$ ) compared to healthy volunteers $(42.27 \mathrm{pg} / \mathrm{ml}, \mathrm{n}=60)$ and the difference was statistically significant $(p=0.01)$.

The genotype frequencies of our population respected Hardy-Weinberg equilibrium distribution both in patients by primary SS than in controls. In PSS patients, the genotype frequencies of $-592 \mathrm{C} / \mathrm{A}$ are $53 \%$ for the CC genotype, $41 \%$ for the $\mathrm{CA}$ and $6 \%$ for the AA genotype. In controls these frequencies are respectively $60.3 \%, 32.9 \%$ and $6.8 \%$. The genotype frequencies of $-1082 \mathrm{G} / \mathrm{A}$ are $29.6 \%$ for the AA genotype, $63 \%$ for the $A G$ and $7.4 \%$ for the GG genotype. In controls these frequencies are respectively $41.5 \%, 52.1 \%$ and $6.3 \%$.

The genotype frequencies of $-819 \mathrm{C} / \mathrm{T}$ are $47.6 \%$ for the CC genotype, $43.9 \%$ for the CT and $8.5 \%$ for the TT genotype. In controls these frequencies are respectively $41.5 \%, 52.1 \%$ and $6.3 \%$.

No significant differences in genotypic frequencies were observed between cases and controls in the three polymorphisms.
Statistical analysis preformed revealed that there was neither protective nor aggravating hapoltype. However ATC haplotype seems to have a protective impact in controls $(p=0.06$ and $O R=0.20)$

Conclusions: IL10 level was significantly higher in PSS patients in precedent studies (1) (2). In our case II10 level was associated with PSS in Tunisian patients but it was statistically lower than controls. Our results show that the three polymorphisms of gene of IL-10 are not a marker of SGS in the Tunisian population. This result might be explained by allelic variation or ethnic group. References:

[1] Marka M, Bessenyei B, Zeher M, Semsei I. IL-10 promoter -1082 polymorphism is associated with elevated IL-10 levels in control subjects but does not explain elevated plasma IL-10 observed in Sjogren's syndrome in a Hungarian cohort. Scandinavian journal of immunology. 2005;62(5):474-80.

[2] Vazquez-Villamar M, Palafox-Sanchez CA, Munoz-Valle JF, Valle Y, OrozcoBarocio G, Hernandez-Bello J, et al. Analysis of IL10 haplotypes in primary Sjogren's syndrome patients from Western Mexico: Relationship with mRNA expression, IL-10 soluble levels, and autoantibodies. Human immunology. 2015;76(7):473-9.

Disclosure of Interest: None declared

DOI: 10.1136/annrheumdis-2017-eular.6617

\section{FRI0291 CD4 AND CD8 COUNT IN PATIENTS WITH SYSTEMIC LUPUS ERYTHEMATOSUS - ASSOCIATIONS WITH INFECTION AND DISEASE ACTIVITY}

H. Baharuddin $^{1}$, N. Zainudin ${ }^{2}$, A. Ahmad ${ }^{2}$, A. Rosman ${ }^{2}$, M. Mohd Zain ${ }^{2}$.

${ }^{1}$ Rheumatology, Universiti Teknologi MARA; ${ }^{2}$ Rheumatology, Hospital Selayang, Selangor, Malaysia

Background: Systemic lupus erythematosus (SLE) is an autoimmune disease driven by the activation of autoreactive T and B cells. Decreased levels of CD4 counts were found in these patients, although there are conflicting data on whether CD4 is associated with SLE disease activity. ${ }^{1,2,3}$

Objectives: To investigate the associations between CD4 and CD8 counts with (1) active SLE and (2) infection in patients with SLE.

Methods: This was a cross-sectional study conducted in a rheumatology referral centre in Malaysia. Inclusion criteria included patients who fulfilled SLICC SLE classification and was admitted to rheumatology ward for active SLE or infection. We excluded patients who had overlap syndrome, retroviral disease or underlying malignancy. SLE Disease Activity Index (SLEDAI) score was assessed by the same rheumatologist.

Statistical analysis was performed using SPSS 20 and a p-value of $<0.05$ was considered significant. Pearson correlation was used to analyse associations between 2 continuous variables, while comparison between 2 continuous data was performed using student's t test.

Results: Forty-two patients participated in this study. Majority $(90.5 \%)$ were females with a mean age of $33.05( \pm 11.42)$ years and SLE duration of 8.69 $( \pm 5.26)$ years. There were $40(95.2 \%)$ ANA positive, $23(54.8 \%)$ anti-dsDNA positive, $13(31.0 \%)$ anti-smith positive, 24 (57.1\%) low C3 and $16(38.1 \%)$ low C4 in this cohort.

There were significant correlations between SLEDAI score and CD4 ( $r=-0.59$, $p<0.01)$ and CD8 counts $(r=-0.57, p<0.01)$. CD4 and CD8 counts were significantly lower in patients with clinically active SLE vs non-active, however, there were no significant differences in CD4 and CD8 counts in patients with infection vs no infection. We also found significantly high anti-dsDNA level and low complement 3 , but not complement 4 , in active SLE. White cell count was significantly higher in patients with infection. The details are tabulated in Table 1.

Table 1. CD4, CD8 and other parameters in infection and active SLE

\begin{tabular}{|c|c|c|c|c|c|c|}
\hline \multirow[t]{2}{*}{ Parameters } & \multicolumn{3}{|c|}{ Infection } & \multicolumn{3}{|c|}{ Active SLE } \\
\hline & Yes $( \pm S D), n=21$ & No ( $\pm S D), n=21$ & $\mathrm{p}$ & Yes $( \pm S D), n=26$ & No ( $\pm S D), n=16$ & $\mathrm{p}$ \\
\hline CD4 & $566.05(482.51)$ & $510.43(479.08)$ & 0.71 & $375.58(348.07)$ & $802.56(544.34)$ & $<0.01$ \\
\hline CD8 & 659.10 (562.91) & $456.48(221.86)$ & 0.13 & $406.0(346.04)$ & $804.44(460.04)$ & $<0.01$ \\
\hline SLEDAI & 7.14 (7.79) & $6.38(7.13)$ & 0.74 & $10.0(7.54)$ & $1.5(3.30)$ & $<0.01$ \\
\hline Anti-dsDNA & $110.17(145.84)$ & $103.14(186.87)$ & 0.91 & $149.01(193.48)$ & $42.47(53.62)$ & 0.05 \\
\hline C3 & $0.85(0.47)$ & 0.93 & 0.56 & $0.78(0.39)$ & $1.06(0.38)$ & 0.03 \\
\hline C4 & $0.21(C$ & 0.19 & 0.53 & $0.19(0.12)$ & 0.21 & 0.56 \\
\hline WBC & 8.92 (3.98) & $6.41(2.52)$ & 0.02 & 7.52 (3.72) & $7.90(3.30)$ & 0.74 \\
\hline
\end{tabular}

Conclusions: There were significant negative correlations between SLEDAI score and CD4 and CD8 counts, with low CD4 and CD8 counts found in patients with active SLE. There were no significant differences in CD4 and CD8 counts during infection.

\section{References:}

[1] Ferreira S, Vasconcelos J, Marinho A et al. CD4 lymphocytopenia in systemic lupus erythematosus. Acta Rheumatol Port 2009; 34: 200-206.

[2] Bonelli M, Savitskaya A, von Dalwigk K et al. Quantitative and qualitative 